\title{
Cavity Formation in Confined Growing Crystals
}

\author{
Felix Kohler, ${ }^{1, *}$ Luca Gagliardi, ${ }^{2, \dagger}$ Olivier Pierre-Louis, ${ }^{2}$ and Dag Kristian Dysthe ${ }^{1}$ \\ ${ }^{1}$ Physics of Geological Processes, The Njord Centre, Department of Geosciences, University of Oslo, Oslo 0316, Norway \\ ${ }^{2}$ Institut Lumière Matière, UMR5306 Université Lyon 1-CNRS, Université de Lyon 69622 Villeurbanne, France
}

(Received 18 January 2018; published 30 August 2018)

\begin{abstract}
Growing crystals form a cavity when placed against a wall. The birth of the cavity is observed both by optical microscopy of sodium chlorate crystals $\left(\mathrm{NaClO}_{3}\right)$ growing in the vicinity of a glass surface, and in simulations with a thin film model. The cavity appears when growth cannot be maintained in the center of the contact region due to an insufficient supply of growth units through the liquid film between the crystal and the wall. We obtain a nonequilibrium morphology diagram characterizing the conditions under which a cavity appears. Cavity formation is a generic phenomenon at the origin of the formation of growth rims observed in many experiments, and is a source of complexity for the morphology of growing crystals in natural environments. Our results also provide restrictions for the conditions under which compact crystals can grow in confinement.
\end{abstract}

DOI: 10.1103/PhysRevLett.121.096101

In natural environments, confinement commonly constrains the growth of crystals [1]. Constrained growth may cause large forces such as in salt weathering [2-5], in the opening of veins in Earth's crust [6,7], or in frost heave $[8,9]$. In biomineralization - the process by which living organisms grow minerals, confinement also plays a key role in controlling the shape and phase of nanocrystals [10,11], and combines with the chemical environment [12] to govern microstructure formation in, e.g., bones or dentine. Beyond its relevance for natural environments, motion produced by confined growth can be used in technological applications such as nanomotors [13]. However, while the morphology of freely growing crystals has been investigated for decades [14,15], much less is known about crystal morphological evolution in confinement. Here, we show that the simplest confinement, i.e., the vicinity of a flat impermeable substrate, leads to the formation of a cavity (or pit) in the growing crystal. The cavity forms due to insufficient material supply in the center of the contact. After their formation, cavities can expand up to the edge of the contact, leading to growth rims that have been observed in force of crystallization experiments since the beginning of the 20th century [16-19].

Cavity formation is observed both using optical microscopy of sodium chlorate crystals $\left(\mathrm{NaClO}_{3}\right)$ growing in the vicinity of a glass surface, and in simulations based on a thin film model. The birth of the cavity is characterized by a nonequilibrium morphology diagram describing the balance between growth rate and mass supply. This diagram is found to be robust with respect to variations in the properties of the growth mechanism such as anisotropy or kinetics. Indeed, despite their differences, e.g., with respect to crystalline anisotropy, both experiments and simulations collapse on the same diagram. Our results provide generic conditions for growing compact crystals without cavities in micro- and nanoconfinement conditions such as those encountered in Earth's crust, in biomineralization, or in technological applications.

Experimental methods and observation of the cavity.-In our experiments, we control the solution supersaturation while measuring the confined crystal topography. A $\mathrm{NaClO}_{3}$ seed crystal with a volume of $\sim 1 \mathrm{~mm}^{3}$ is placed in a $60 \mu \mathrm{l}$ chamber filled with a saturated $\mathrm{NaClO}_{3}$ solution, and is then dissolved to the desired size. The solubility $c_{0}(T)$ of $\mathrm{NaClO}_{3}$ is strongly temperature dependent [20-22]. The temperature of the sample chamber and oil immersion objective is controlled with a long term precision of $1 \mathrm{mK}$. By adjusting the temperature $T$ below or above the equilibrium temperature $T_{\text {eq }}$, to obtain growth or dissolution, the relative saturation $\sigma_{b}=\left(c_{b}-c_{0}(T)\right) / c_{0}(T)$ can be controlled with an accuracy of $0.1 \%$. The equilibrium point, $c_{b}=c_{0}\left(T_{e q}\right)$, is identified when the crystal exhibits roundish edges and neither grows nor dissolves. The high nucleation barrier of $\mathrm{NaClO}_{3}$ prevents the appearance of other seed crystals in the chamber that could affect the concentration of the bulk solution [23]. Additional details about preparation of seeds and temperature control are provided in the Supplemental Material [24].

The confined (100) facet of the crystal is observed from below using reflection interference contrast microscopy (RICM), which is based on the interference between reflections from the glass interface and the confined crystal interface (see Fig. 1). Using a specialized objective, a high power LED light source and a 16 bit camera this method allows us to determine the distance $\zeta(\mathbf{r})$ between the crystal and the glass with $\mathrm{nm}$ precision [30]. Because of the presence of dust grains on the substrate, the distance $\zeta(\mathbf{r})$ cannot be decreased below a minimum value, which ranges 


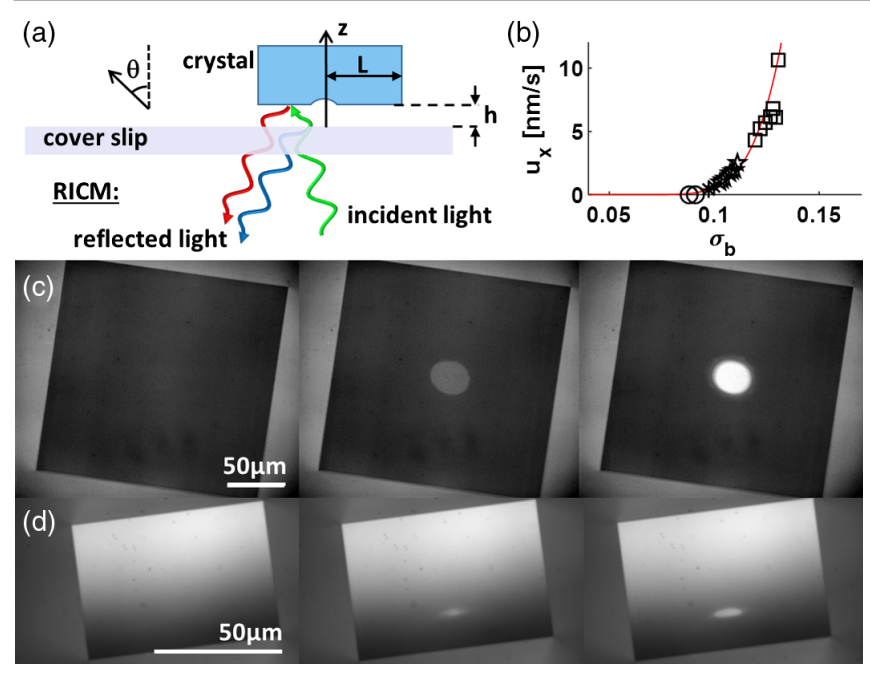

FIG. 1. Experimental setup and observation of cavities. (a) A growing crystal is placed against a glass substrate. The crystal surface profile is determined with $\mathrm{nm}$ accuracy by RICM (see text). (b) Measured growth rate $u_{x}$ of lateral facets as function of bulk supersaturation $\sigma_{b}$. Different symbols correspond to distinct measurement series. Red line: fit according to the nucleationlimited growth rate [31]. (c) RICM images showing the formation of a cavity in the (100) facet during growth. Snapshots just before the start of cavity formation, 15 min later, and 35 min later. Supersaturation: $\sigma_{b}=0.093$, distance to the glass substrate: $h=51 \mathrm{~nm}$. (d) Same as (c) for an inclined crystal, supersaturation: $\sigma_{b}=0.21$, distance to the glass substrate: 12 (bottom edge) to $95 \mathrm{~nm}$ (top edge).

from 10 to $80 \mathrm{~nm}$. An atomic force microscope image of the substrate showing these dust grains is provided in the Supplemental Material [24]. In order to gain more control on the gap between the substrate and the crystal we have also performed experiments with glass beads deposited on the substrate prior to the seed crystal, which act as calibrated spacers (see the Supplemental Material [24] for details). In all measurements, the lateral extent $2 L$ of the crystal facet facing the substrate is determined by tracking the edges with a precision of $15 \mathrm{~nm}$. The growth rate $u_{x}=\dot{L}$ of lateral facets shown in Fig. 1(b), which is nonvanishing only above a critical supersaturation $\sim 0.1$, is characteristic of growth limited by two-dimensional nucleation [31]. In rare cases, the saturation dependence of $u_{x}$ deviated from this characteristic behavior due to the presence of dislocations. Such cases were discarded, and all the results reported below therefore correspond to dislocation-free experiments.

Our main observation is that during growth when the size $2 L$ exceeds a critical value, which depends on the average film width $h$ and on the supersaturation $\sigma_{b}$, a cavity forms within the contact region. Snapshots of the temporal evolution slightly above the threshold are shown in Fig. 1(c). The corresponding surface plots and movie are respectively shown in Fig. 2(a), and the Supplemental Material [32]. The appearance of the cavity can be interpreted as a consequence of a lower growth rate in the central part of the facet as compared to the parts closer to the facet edges. Intuitively, this lower growth rate is due to confinement limiting the diffusive mass supply from the bulk liquid.

Simulation model and observation of the cavity.-In parallel, we have performed numerical simulations of a thin film model describing the dynamics within the contact region. The model, based on that of Refs. [33,34], describes growth and dissolution of a crystal, considered as a rigid body without elastic deformation, coupled to diffusion and hydrodynamics in the liquid film. We used some additional simplifying assumptions. First, attachment-detachment kinetics of ions are fast at the surface of salts such as $\mathrm{NaClO}_{3}$, and diffusion limited mass transport along the thin liquid film is decreased by confinement. Hence, we can safely assume that kinetics are limited by diffusion (see the Supplemental Material for a discussion [24]). Moreover, whereas the bulk solution surrounding the crystal is influenced by solutal buoyancy convection, which originates from temperature and concentration gradients, such effects can be excluded in the confined solution below the crystal. We also neglect the hydrodynamic flow induced by the density difference between the crystal and the solution during growth [35], though, we keep the density difference as the origin of the gravitational force $F_{z}$ maintaining the crystal on the substrate. The model is axisymmetric about the $\mathbf{z}$ axis defined in Fig. 1.

Using the small slope limit and the dilute limit [33], we then obtain two equations accounting for the evolution of the local thickness $\zeta(r, t)$ of the liquid film where $r$ is the radial coordinate, and for the growth rate $u_{z}(t)$, which is the velocity of the crystal along $\mathbf{z}$. The first equation accounts for local mass balance, the second for global force balance

$$
\begin{gathered}
\partial_{t} \zeta=-\frac{\Omega D}{r} \partial_{r}\left[r \zeta \partial_{r} c(r)\right]-u_{z}, \\
c(r)=c_{0}\left(1+\frac{\Omega}{k_{B} T}\left[\tilde{\gamma} \partial_{r r} \zeta+\frac{\tilde{\gamma}}{r} \partial_{r} \zeta-U^{\prime}(\zeta)\right]\right), \\
u_{z} 2 \pi \int_{0}^{R} d r r \int_{r}^{R} d r^{\prime} \frac{6 \eta r^{\prime}}{\zeta\left(r^{\prime}\right)^{3}}=F_{z}+2 \pi \int_{0}^{R} d r r U^{\prime}(\zeta),
\end{gathered}
$$

where we have defined the viscosity $\eta$, diffusion constant $D$, molecular volume $\Omega$, numerical concentration $c(r)$, and solubility $c_{0}$, Boltzmann constant $k_{B}$, and temperature $T$. The interaction between the substrate and the crystal in Eq. (1) is described by the potential $U(\zeta)$. In order to mimic the experimental conditions where thicknesses smaller than $h$ are forbidden by dust grains, we consider the repulsive potential

$$
U(\zeta)=\mathcal{A} f\left(\frac{\zeta-h}{\bar{\lambda} h}\right)
$$




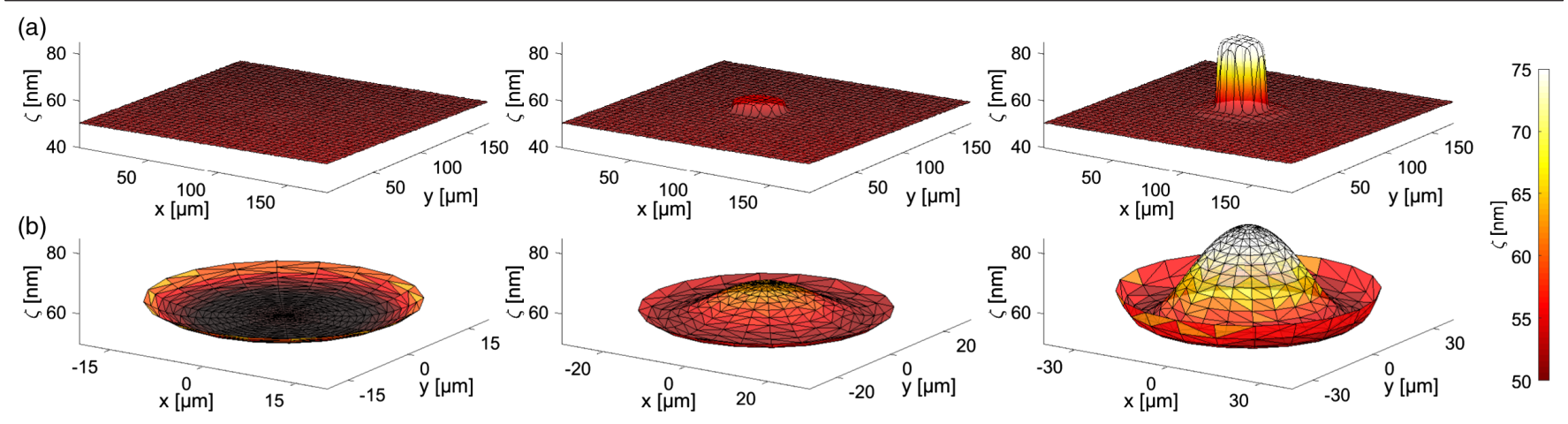

FIG. 2. 3D view of cavity formation at the confined crystal interface. (a) Surface plot of the distance $\zeta(\mathbf{r})$ between crystal interface and glass substrate from the RICM images reported in Fig. 1(c). (b) Simulation result showing axisymmetric steady states with film width $h=50 \mathrm{~nm}$, supersaturation $\sigma_{\mathrm{BC}} \approx 0.004$ and thickness $\zeta_{\mathrm{BC}}=1040 \mathrm{~nm}$ at the edge of the simulation box of radius $R$. The surface plots represent only the contact region of radius $L<R$ with supersaturation $\sigma_{b}=\sigma(L)<\sigma_{\mathrm{BC}}$ at their edge. From left to right: $R=60 \mu \mathrm{m}$, $L \approx 20 \mu \mathrm{m}$, and $\sigma_{b} \approx 0.0011 ; R=65 \mu \mathrm{m}, L \approx 28 \mu \mathrm{m}$, and $\sigma_{b} \approx 0.0014 ; R=70 \mu \mathrm{m}, L \approx 37 \mu \mathrm{m}$, and $\sigma_{b} \approx 0.0019$.

where $\mathcal{A}$ and $\bar{\lambda}$ are constants, and $f(x)=e^{-x} / x$ is a Yukawa-like term. Finally, we have defined the surface stiffness $\tilde{\gamma}=\gamma(0)+\gamma^{\prime \prime}(0)$ in Eq. (1), where $\gamma(\theta)$ is the surface free energy and the angle $\theta$ is defined in Fig. 1.

In experiments, the crystal surface facing the substrate is a facet, and surface stiffness is expected to diverge for faceted orientations while surface tension remains finite [14], leading to a singular crystal shape. Such singularities cannot be handled by our continuum model where the crystal surface exhibits a smooth nonsingular profile. However, we approach the behavior of a facet using a strongly anisotropic model with a stiffness $\tilde{\gamma}=10^{2} \mathrm{~J} / \mathrm{m}^{2}$, roughly $10^{3}$ times larger than the expected surface tension $\gamma(0) \sim$ $0.1 \mathrm{~J} / \mathrm{m}^{2}$ (see the Supplemental Material [24] for a detailed discussion). Choosing the other model parameters in a way which is consistent with the literature and with experiments (see the Supplemental Material [24]), this assumption on the stiffness allows one to obtain growth rates and supersaturations comparable to those observed in experiments.

We numerically solved Eqs. 1(a), 1(b) in a circular simulation box of fixed radius $R$, with fixed film width $\zeta(R)=\zeta_{\mathrm{BC}}$ and supersaturation $\sigma(R)=\sigma_{\mathrm{BC}}$ at the boundary of the integration domain. All simulations were started with a flat contact region.

Steady-state profiles are reached at long simulation times. They are reported in Fig. 2(b) for increasing sizes $R$ of the simulation box. A movie of the related time evolution is reported in the Supplemental Material [36]. As in the experiments, we find that a cavity forms when the size of the crystal exceeds a critical value. As shown in Fig. 2(b), the effective radius $L$ of the contact is smaller than the total radius $R$ of the simulation box. Despite the absence of growth-induced expansion of the contact size $L$ in simulations, good qualitative agreement is obtained with the experiments. This agreement suggests a quasistatic behavior, where the growth of the lateral crystal size is slow enough to have a negligible influence on the diffusion field in the contact region.
Criterion for cavity formation.-Based on this hypothesis of quasistatic dynamics, the threshold for cavity formation can be deduced from global mass conservation. Within the thin film approximation, the concentration does not depend on the $z$ coordinate, and mass balance for a disc of radius $r$ and constant thickness $h$ of liquid film centered in the contact region is obtained from the substitution $\zeta=h$ and $\partial_{t} \zeta=0$ in Eq. 1(a), leading to

$$
\pi r^{2} J_{k}=-2 \pi r h J_{d}(r)
$$

where $J_{k}=u_{z} / \Omega$ is the mass flux entering the crystal per unit facet area, and $J_{d}(r)=-D(d c / d r)$ denotes the diffusion flux entering into the liquid volume. The concentration is integrated as

$$
c(r)=c_{b}-\frac{J_{k}}{4 h D}\left(L^{2}-r^{2}\right),
$$

where $c_{b}$ is the concentration at the edge of the contact region. The local supersaturation $\sigma(r)=c(r) / c_{0}-1$ decreases toward the center of the facet. We expect that growth can be maintained in the central region only if the supersaturation is positive at $r=0$. More precisely, from Eq. (1b), we infer that since cavity formation requires the concomitant cancellations of the local curvature $\partial_{r r} \zeta+\partial_{r} \zeta / r$ to form a concave part in the center, and of the substrate repulsion $U^{\prime}(\zeta)$ since interactions vanish when the interface moves away from the substrate, it should correspond to the cancellation of the supersaturation at $r=0$. This is confirmed by the numerical solution of Eq. (1) showing that a cavity starts forming approximately when $\sigma(0)=0$. This condition for cavity formation can be rewritten as

$$
u_{z} \geq 4 \Omega c_{0} \sigma_{b} D \frac{h}{L^{2}}=\beta D,
$$

where $\beta=4 \Omega c_{0} \sigma_{b} h / L^{2}$, and $\sigma_{b}=\sigma(L)=c_{b} / c_{0}-1$.

Simulation morphology diagram.-Simulation results reported in the $\left(u_{z} / D, \beta\right)$ plane in Fig. 3(b) confirm the 

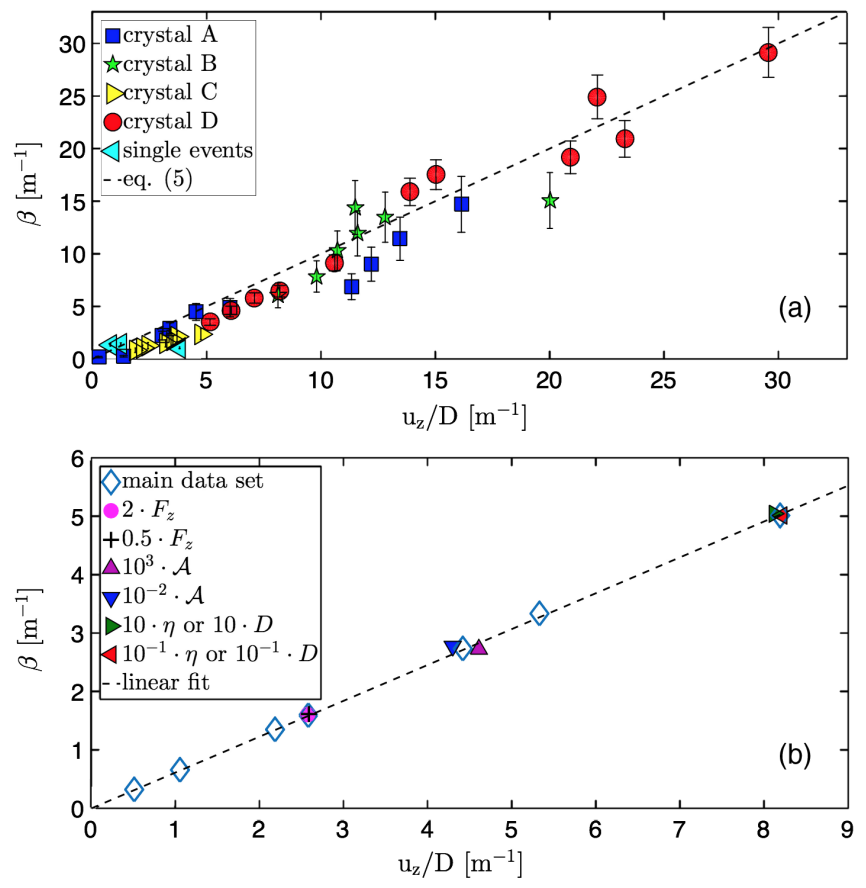

FIG. 3. Nonequilibrium morphology diagram for cavity formation. (a) Transition line in experiments for different crystals. Results plotted assuming $D=0.0935 \times 10^{-9} \mathrm{~m}^{2} \mathrm{~s}^{-1}$ which allows for a perfect correspondence with Eq. (5). (b) Transition line obtained from simulations. Colored filled dots were obtained using different values of the repulsion strength $\bar{a}$, viscosity $\eta$, or diffusion $D$ and external force $F_{z}$, with respect to the main set of simulations.

prediction of Eq. (5). However, the slope $D \beta / u_{z} \approx 0.61$ is slightly lower than the expected value $D \beta / u_{z}=1$. Details on the methods to determine the transition point, the contact size $L$, and supersaturation $\sigma_{b}=\sigma(L)$ at the edge of the contact region are reported in the Supplemental Material [24]. We have checked that these conclusions are not affected by the boundary conditions imposed for numerical integration. Since the quantity $\beta$ depends on $h$, the dimensionless range $\bar{\lambda}$ of the repulsion potential is kept very small $\sim 10^{-2}$, so that the liquid film thickness in the stable regime is approximately equal to $h$ in all simulations.

One striking property of the transition line is its robustness with respect to the variation of the physical parameters that do not enter into Eq. (5). Indeed, as shown in Fig. 3(b), large variations in gravitational force $F_{z}$, and normalized interaction amplitude $\mathcal{A}$, lead to negligible changes in the transition line position. Furthermore, increasing or decreasing one of the kinetic constants $D$ or $\eta$ by a factor of 10 also does not affect the transition line.

Experimental morphology diagram.-In experiments, we have performed growth cycles to explore a range of supersaturations with a single sample. For each cycle, we monitored the surface profile $\zeta(\mathbf{r})$ during growth at fixed supersaturation, and recorded the critical size at which the cavity forms. As soon as the depth of the cavity exceeded
$15 \mathrm{~nm}$, the temperature was increased to attain a saturation value at which the cavity closes again. Once we obtained a flat interface, the entire procedure was automatically repeated with a different growth supersaturation. The vertical growth rate $u_{z}$ is obtained from the increase of the depth of the cavity just after its formation. This method assumes that the growth rate at the bottom of the cavity is negligible leading to a deepening which is only due to the growth rate $u_{z}$ of the contact region outside the cavity. Since the lateral growth rate $u_{x}$ is easier to determine than $u_{z}$ from the growth of the cavity, we measure $u_{x}$, and determine $u_{z}$ from a linear interpolation of the relation between the two velocities based on a large number of measurements. The ratio $u_{x} / u_{z}$ is roughly independent of $L, h$, and $\sigma_{b}$, as shown in the Supplemental Material [24].

Our measurements reported in Fig. 3(a) agree with a linear behavior of the transition line in the $\left(u_{z} / D, \beta\right)$ plane. Diffusion constants $D=0.093 \times 10^{-9} \mathrm{~m}^{2} \mathrm{~s}^{-1}$ or $D=0.057 \times 10^{-9} \mathrm{~m}^{2} \mathrm{~s}^{-1}$, respectively, provide quantitative agreement with the slopes predicted by Eq. (5) or by simulations in Fig. 3(b). These constants are consistent with values reported in the literature [37].

Geometrical corrections described in the Supplemental Material [24] were used to evaluate $\beta$ for elongated and inclined crystals in Fig. 3(a). Moreover, for inclined crystals, the minimum of supersaturation where the cavity should form is found to be shifted toward the side where the film is thinner due to the reduced mass transport in this part. The expression of this shift is calculated in the Supplemental Material [24]. In Fig. 1(d), the observed ratio of the distance from the lower edge to the width of the facet is $0.254 \pm 0.005$, in reasonable agreement with the prediction $0.32 \pm 0.05$ calculated from the expression given in the Supplemental Material [24], using the experimentally measured inclination.

Discussion.-The dependence of $u_{z}$ on physical parameters is different in simulations and experiments. For example, while $u_{z}$ is roughly independent of $h$ in experiments, it is proportional to $h$ in simulations. Despite this difference, simulations and experiments fall in the same morphology diagram in the $\left(u_{z} / D, \beta\right)$ plane.

Additional differences between experiments and simulations have been observed. First, the shape of the cavity is less rounded in experiments, and emerges from a flat surrounding facet, as seen in Fig. 2(a). This is a consequence of the difference between crystalline anisotropy in experiments giving rise to facets and to large slope variations, and our model anisotropy without singular facets and within the small slope approximation. In order to reproduce the details of the anisotropy of the experimental shape, one promising strategy is to describe explicitly the dynamics of atomic steps, as suggested by previous studies of facet instabilities during free growth $[38,39]$. Second, close to the threshold, random opening and closure of the cavity are observed in experiments. The results reported above correspond to the 
lower boundary of the stochastic transition regime. Such fluctuations could be attributed to a nucleationlike process associated to the competition between thermal fluctuations, and surface tension driven decay of the cavity. Again, despite these differences, both experiments and simulations collapse on a linear transition line in the $\left(u_{z} / D, \beta\right)$ plane. The robustness of this linearity can be traced back to the fact that it depends only on two ingredients: mass conservation, and diffusion-limited mass transport, as discussed above in the derivation of Eq. (5).

In conclusion, when a growing crystal is placed in the vicinity of a flat wall, a cavity can form in the surface of the crystal facing the wall. The presence of a cavity can be predicted from the crossing of a linear transition line in the $\left(u_{z} / D, \beta\right)$ plane. Cavity formation in confinement appears as an alternative path toward the formation of concave crystals, beyond well known free growth instabilities leading, e.g., to dendrites [40] or hopper crystals [38,41].

In later stages of growth the cavity can expand up to the edges of the contact area, leading to a growth rim, as observed in force of crystallization experiments $[16,17]$. Since the birth of the cavity affects the shape and area of the contact, cavity formation should also influence the force and interactions between the crystal and its environment.

This project has received funding from the European Union's Horizon 2020 research and innovation programme under the Marie Sklodowska-Curie Grant Agreement No. 642976 (ITN NanoHeal) and from the Norwegian Research Council Grant No. 222386.

*felixkohler@gmail.com †luca.gagliardi@univ-lyon1.fr

[1] C. Alba-Simionesco, B. Coasne, G. Dosseh, G. Dudziak, K. E. Gubbins, R. Radhakrishnan, and M. SliwinskaBartkowiak, J. Phys. Condens. Matter 18, R15 (2006).

[2] A. Goudie and H. Viles, Salt Weathering Hazards (John Wiley \& Sons, 1997), p. 256.

[3] L. A. Rijniers, H. P. Huinink, L. Pel, and K. Kopinga, Phys. Rev. Lett. 94, 075503 (2005).

[4] R. J. Flatt, F. Caruso, A. A. M. Sanchez, and G. W. Scherer, Nat. Commun. 5, 4823 (2014).

[5] A. Naillon, P. Joseph, and M. Prat, Phys. Rev. Lett. 120, 034502 (2018).

[6] D. V. Wiltschko and J. W. Morse, Geology 29, 79 (2001).

[7] J. P. Gratier, E. Frery, P. Deschamps, A. Røyne, F. Renard, D. Dysthe, N. Ellouz-Zimmerman, and B. Hamelin, Geology 40, 1015 (2012).

[8] L. A. Wilen and J. G. Dash, Phys. Rev. Lett. 74, 5076 (1995).

[9] A. W. Rempel, J. S. Wettlaufer, and M. G. Worster, Phys. Rev. Lett. 87, 088501 (2001).

[10] B. Cantaert, E. Beniash, and F. C. Meldrum, Chemistry 19, 14918 (2013).

[11] Y.-W. Wang, H. K. Christenson, and F. C. Meldrum, Chem. Mater. 26, 5830 (2014).
[12] M. Tanaka, E. Mazuyama, A. Arakaki, and T. Matsunaga, J. Biol. Chem. 286, 6386 (2011).

[13] B. C. Regan, S. Aloni, K. Jensen, R. O. Ritchie, and A. Zettl, Nano Lett. 5, 1730 (2005).

[14] Y. Saito, Statistical Physics of Crystal Growth (World Scientific, Singapore, 1996).

[15] A. Pimpinelli and J. Villain, Physics of Crystal Growth (Cambridge University Press, Cambridge, England, 1998).

[16] G. F. Becker and A. L. Day, Proceedings of the Washington Acadamy of Science VII, 283 (1905).

[17] G. F. Becker and A. L. Day, Note on the Linear Force of Growing Crystals (The University of Chicago Press, 1916), Vol. 24, pp. 313.

[18] P. K. Weyl, J. Geophys. Res. 64, 2001 (1959).

[19] A. Røyne and D. K. Dysthe, J. Cryst. Growth 346, 89 (2012).

[20] A. Seidell, Solubilities of Inorganic and Metal Organic Compounds (New York, Van Nostrand, 1919), p. 904.

[21] B. Crump, M. F. Hoq, B. Indu, and W. R. Emst, J. Chem. Eng. Data 40, 1106 (1995).

[22] C. C. Kerr-McGee, The Chlorate Manual (Kerr-McGee Chemical Corp., Oklahoma, 1985).

[23] R. Y. Qian and G. D. Botsaris, Chem. Eng. Sci. 53, 1745 (1998).

[24] See Supplemental Material at http://link.aps.org/ supplemental/10.1103/PhysRevLett.121.096101 for details on experiments and numerical methods, which also includes Refs. [25-29].

[25] W. Wang and W. R. Hu, J. Cryst. Growth 160, 398 (1996).

[26] M. Ajitha Sweetly, S. Ramalingam, T. Chithambarathanu, and P. Selvarajan, Int. J. Adv. Sci. Tech. Res. 3, 1042 (2012).

[27] A. Campbell, E. Kartzmark, and B. G. Olivier, Can. J. Chem. 44, 925 (1966).

[28] M. V. Ramana Murty, Phys. Rev. B 62, 17004 (2000).

[29] J.-N. Aqua, A. Gouyé, A. Ronda, T. Frisch, and I. Berbezier, Phys. Rev. Lett. 110, 096101 (2013).

[30] L. Limozin and K. Sengupta, Chem. Phys. Chem. 10, 2752 (2009).

[31] F. Abbona and D. Aquilano, in Springer Handbook of Crystal Growth (Springer Berlin Heidelberg, Berlin, Heidelberg, 2010), p. 53.

[32] See Supplemental Material at http://link.aps.org/ supplemental/10.1103/PhysRevLett.121.096101 for movie showing cavity initiation on the confined $\mathrm{NaClO} 3$ interface.

[33] L. Gagliardi and O. Pierre-Louis, Phys. Rev. E 97, 012802 (2018).

[34] L. Gagliardi and O. Pierre-Louis, New J. Phys. 20, 073050 (2018).

[35] W. R. Wilcox, Prog. Cryst. Growth Characterization Mater. 26, 153 (1993).

[36] See Supplemental Material at http://link.aps.org/ supplemental/10.1103/PhysRevLett.121.096101 for a 3D animation of the numerical solution.

[37] A. N. Campbell and B. G. Oliver, Can. J. Chem. 47, 2681 (1969).

[38] A. Chernov, J. Cryst. Growth 24-25, 11 (1974).

[39] H. Lin, P. G. Vekilov, and F. Rosenberger, J. Cryst. Growth 158, 552 (1996).

[40] J. S. Langer, Rev. Mod. Phys. 52, 1 (1980).

[41] I. Sunagawa, J. Cryst. Growth 99, 1156 (1990). 